\title{
Circuit
}

Musiques contemporaines

\section{La chronologie des oeuvres de Claude Vivier}

Historisation de la déshistoire

\section{A Chronology of Works by Claude Vivier}

\section{Jaco Mijnheer et Thérèse Desjardins}

Volume 2, numéro 1-2, 1991

Claude Vivier

URI : https://id.erudit.org/iderudit/902025ar

DOI : https://doi.org/10.7202/902025ar

Aller au sommaire du numéro

Éditeur(s)

Les Presses de l'Université de Montréal

ISSN

1183-1693 (imprimé)

1488-9692 (numérique)

Découvrir la revue

Citer cet article

Mijnheer, J. \& Desjardins, T. (1991). La chronologie des oeuvres de Claude Vivier : historisation de la déshistoire. Circuit, 2(1-2), 17-30.

https://doi.org/10.7202/902025ar
Résumé de l'article

On ne disposait pas, jusqu'à présent, d'une liste systématique des oeuvres de Vivier établie selon les critères de la musicologie historique : en se fondant sur les manuscrits et de nombreux documents d'archives, les auteurs portent à 49 le nombre d'oeuvres du compositeur et proposent une datation rigoureuse des pièces. 


\section{La chronologie des œuvres de Claude Vivier Historisation de la déshistoire Jaco Mijnheer et Thérèse Desjardins}

Que propose la musique, sinon l'aménagement dans le temps «historique» d'une ouverture vers une autre temporalité? La musique déchire le temps historique et, pour de brefs instants, montre le hors-temps, fluidité équivoque de l'espace musical. La qualité première de la musique c'est justement une absence - absence de liens entre l'instant d'avant et l'instant d'après; [...] Par son exercice elle contribue à la déshistorisation de l'histoire humaine. (Vivier, ici même: "Que propose la musique?», p. 129.)

À première vue, on éprouve un certain malaise à vouloir inscrire sous le régime unidimensionnel du temps linéaire les créations d'un homme qui justement, par le moyen de la composition musicale, s'efforçait d'y échapper. Mais une liste chronologique des œuvres de Claude Vivier est devenue une nécessité au moment où elles commencent à percer à l'échelle mondiale. Pour pouvoir analyser, évaluer ou critiquer l'œuvre de Vivier, nous devons être capables de suivre les étapes de sa production; nous ne pouvons plus nous contenter des listes classant les compositions selon leur instrumentation ou selon l'ordre alphabétique de leurs titres.

De plus, l'accumulation de renseignements contradictoires sur la chronologie des œuvres et des événements biographiques de Vivier demande, dans la mesure du possible, une clarification définitive. D'ailleurs, le principal coupable de cette confusion historique est Claude Vivier lui-même, pour qui l'exactitude de ce genre d'informations, semble-t-il, n'était pas primordiale(1). Mais on ne peut s'attendre à autre chose de celui qui a écrit: "Le temps est un espace à géométrie variable. Les différents plans se côtoient, se pénètrent et se transforment suivant les règles merveilleuses de la mécanique céleste", ("Pour Gödel", ici même, p. 125.) Le présent inventaire chronologique se veut un premier pas dans la préparation du catalogue officiel des œuvres de Vivier.

Nous donnerons tout d'abord; en trois temps; quelques précisions sur le contenu de la liste: une énumération des différentes catégories de don-
(1) Dans le catalogue des œuvres de Claude Vivier, publié par feu la Société de droits d'exécution du Canada (S.D.E.) et établi avec la coopération de Vivier lui-même, plusieurs dates ne correspondent pas à la signature que nous avons trouvée dans les partitions. La même chose est vraie de certaines affirmations faites par lui en entrevue. II est compréhensible que, dans les travaux ultérieurs sur Vivier, de telles erreurs aient été répétées. 
nées, une explicitation des critères de sélection des œuvres et l'identification de quelques problèmes rencontrés lors de l'établissement de la liste. Nous sommes conscients du fait que, à ce stade-ci, elle n'est pas encore aussi complète et surtout aussi précise qu'elle devrait l'être. Il peut sembler curieux de la publier maintenant, mais c'est l'occasion d'inviter nos lecteurs à nous fournir des informations qui nous permettraient de parfaire cette chronologie.

\section{Les données}

Comme notre intention n'est pas de présenter ici un catalogue d'œuvres, nous avons limité les données sur chaque œuvre à: son titre, la date et le lieu de composition; et, sous forme abrégée, l'instrumentation. Pour établir le titre exact d'une œuvre, nous avons respecté le manuscrit de la partition à l'exception de quelques corrections (lettres majuscules en anglais ou en allemand, notamment). Nous avons enlevé les sous-titres spécifiant l'instrumentation («pour piano», par exemple) par souci de cohérence.

En ce qui concerne la date de composition, nous retenons celle où la rédaction a été achevée (jour, mois et année). Normalement, la source de cette information est le manuscrit de la partition. Dans le cas où cette date ne figure pas ou figure seulement en partie sur la partition, nous l'avons établie à partir de déductions qui sont explicitées. Nous sommes bien conscients qu'établir une liste chronologique d'œuvres uniquement sur la base de la date de signatures est un compromis. Pour donner une idée plus précise des activités compositionnelles de Vivier, il faudrait rendre compte de la période durant laquelle il travaillait sur une œuvre, ce qui demanderait des recherches biographiques plus approfondies. Remarquons toutefois que cet angle d'observation ne changerait pas nécessairement l'ordre des œuvres.

Par lieu de composition, nous entendons le nom de la ville inscrite à la signature de la partition. Comme dans le cas précédent, nous avons essayé de le préciser à l'aide d'autres documents, si nécessaire.

Nous ne présentons l'instrumentation que sous forme abrégée pour alléger le texte et pour faciliter la présentation schématique des données. Dans le contexte présent, elles serviront surtout de rappel au lecteur. 


\section{L'établissement du corpus des œuvres}

Notre liste chronologique inclut toutes les œuvres de Claude Vivier connues de nous; nous n'excluons pas d'éventuelles découvertes ultérieures.

Nous considérons comme une "œuvre» toute composition ayant dépassé l'état conceptuel, c'est-à-dire celle dont la rédaction (par écrit ou sur bande) était réellement commencée. Nous n'avons porté aucun jugement de valeur sur les œuvres ni évalué leur cheminement.

La liste commence par un groupe de cinq œuvres soumises au concours de composition du Conservatoire de musique de Montréal en 1971, à la fin des études de Vivier ${ }^{(2)}$. Nous ne disposons pas de renseignements précis sur d'éventuelles œuvres antérieures composées dans le cadre de ses cours.

Au cours de sa carrière, Vivier a pris ses distances par rapport à certaines de ses compositions, au point de les retirer de son catalogue(3). Apparemment, il prenait ces décisions sans être convaincu de leur nécessité esthétique. Elles n'étaient pas très cohérentes car, même s'il avait retiré une œuvre, il ne s'opposait pas aux exécutions postérieures. Nous avons donc jugé bon de négliger cet aspect ambigu, d'autant plus qu'un des buts de notre chronologie est de rendre compte, sous un angle diachronique, de l'activité compositionnelle de Vivier et non de la réception de ses œuvres par d'autres personnes ou par lui-même.

Les œuvres révisées par Vivier (à notre connaissance, il s'agit de Prolifération et de Désintégration) sont incluses deux fois dans la chronologie puisque la révision d'une composition, autant que sa version originale, constitue une véritable étape de l'itinéraire compositionnel.
(2) Vivier a remporté le premier prix, comme l'année précédente au concours d'analyse, avec son travail sur Arcana de Varèse.

(3) Par exemple, une feuille de 1971 jointe aux cinq partitions soumises au concours de composition du Conservatoire de Montréal (archives du Conservatoire de Montréal) précise que, parmi elles, il "ne considère comme OPUS que Prolifération et Hiérophanie».

\section{Problèmes de chronologie}

Notre liste chronologique reflète deux sortes de problèmes dus à un manque de données. Nous ignorions soit la date exacte de composition d'une œuvre particulière, soit, à l'intérieur de certaines limites, les dates de plusieurs œuvres qui se suivent. Ainsi nous nous trouvions, dans le premier cas, devant des possibilités multiples de situer l'œuvre en question ou, dans le deuxième cas, devant la permutabilité d'une suite d'œuvres. 
Pour résoudre le premier problème, nous avons choisi la représentation suivante:
41. Cinq Chansons pour percussion
42. Prologue pour un Marco Polo
43. Wo bist du Licht!
$<44>$ Bouchara $<42-45>$
45. Samarkand
46. Et je reverrai cette ville étrange

Cet extrait de la liste se lit ainsi. L'ordre des œuvres numérotées 41 , $42,43,45$ et 46 est connu : nous savons avec certitude que Prologue pour un Marco Polo a été signé postérieurement à Cinq Chansons pour percussion et antérieurement à Wo bist du Licht!, etc. Par contre, la position de Bouchara n'est pas certaine, d'où les soufflets. Il est probable que Bouchara ait été composée entre les œuvres 43 et 45 , mais la marge d'hésitation s'étend du numéro 42 au numéro 45 inclusivement. En d'autres mots, nous ne voulons pas exclure, sur la base des données présentement disponibles, que Bouchara se situe immédiatement avant Prologue pour un Marco Polo ou après Samarkand.

En ce qui concerne le deuxième problème, c'est-à-dire la permutabilité des œuvres au sein d'une série, nous le représentons comme suit:

$$
\begin{aligned}
11 . & \text { Désintégration } \\
<12> & \text { Chants } \\
<13> & \text { O! Kosmos } \\
<14> & \text { Jesus erbarme dich } \\
& \text { Désintégration (rév.) } \\
15 . & \text { Lettura di Dante }
\end{aligned}
$$

On voit ici qu'entre la première version et la révision de Désintégration, Vivier a produit trois œuvres, les numéros 12, 13 et 14 de la liste, dont nous ignorons l'ordre. Il se peut, par exemple, que Jesus erbarme dich soit antérieur à Chants et que Chants soit postérieur à O! Kosmos.

Finalement, il arrive que les deux problèmes surviennent en même temps, comme c'est le cas avec la production de l'année 1975.

On trouvera ci-après notre liste chronologique, suivie d'une explicitation des sources pour chaque œuvre. La signature entre guillemets respecte la ponctuation, les abréviations ef l'orthographe de Vivier. Les données entre crochets, bien qu'elles ne soient pas confirmées sans équivoque par aucun document original, sont fort probablement correctes. Toutes les sources dont la référence complète n'est pas fournie se trouvent aux archives des Amis de Claude Vivier. 


\begin{tabular}{|c|c|c|c|}
\hline 1. Quatuor à cordes & [1968] & [Montréal] & 2 vlns, alto, vic. \\
\hline 2. Ojikawa & [1968] & [Montréal] & Sop., clar., perc. \\
\hline $\begin{array}{l}\text { 3. Musique pour } \\
\text { une liberté à bâtir }\end{array}$ & [1968 ou 1969] & [Montréal] & $\begin{array}{l}\text { Chœur SA, } \\
\text { grand orchestre }\end{array}$ \\
\hline 4. Prolifération & [1969] & [Montréal] & $\begin{array}{l}\text { Ondes Martenot, } \\
\text { pno, perc. }\end{array}$ \\
\hline 5. Hiérophanie & 8-1-197 & Montréal & $\begin{array}{l}\text { Sop., vents, } \\
2 \text { perc. }\end{array}$ \\
\hline $\begin{array}{l}\text { 6. Musik für } \\
\text { das Ende }\end{array}$ & $7-1971$ & Cologne & 20 voix mixtes \\
\hline 7. Deva et Asura & $8-1-1972$ & Utrecht & $\begin{array}{l}15 \text { vents, } \\
5 \text { cordes }\end{array}$ \\
\hline 8. Variation I & c. $2-1972$ & Utrecht & Bande \\
\hline 9. (sans titre) (4) & $10-3-1972$ & Utrecht & Bande \\
\hline $\begin{array}{l}\text { 10. Hommage. Musique } \\
\text { pour un vieux Corse triste }\end{array}$ & c. $5-1972$ & Utrecht & Bande \\
\hline 11. Désintégration & $26-8-1972$ & Paris & 2 pnos \\
\hline$<12>$ Chants & [1973] & Cologne & $\begin{array}{l}2 \text { sop., } \\
2 \text { mezzo-sop., } \\
3 \text { contraltos }\end{array}$ \\
\hline$<13>0$ ! Kosmos & $13-2-1973$ & Cologne & Chœur SATB \\
\hline $\begin{array}{l}<14>\text { Jesus } \\
\text { erbarme dich }\end{array}$ & [1973] & [Cologne] & Chœur SATB \\
\hline $\begin{array}{l}\text { Désintégration } \\
\text { (rév.) }\end{array}$ & $3-4-1974$ & Cologne & $\begin{array}{l}2 \text { pnos, } 6 \\
\text { cordes }\end{array}$ \\
\hline 15. Lettura di Dante & $16-7-1974$ & Cologne & $\begin{array}{l}\text { Sop., petit } \\
\text { ensemble }\end{array}$ \\
\hline $\begin{array}{l}\text { 16. Pièce pour } \\
\text { flûte et piano }\end{array}$ & $16-2-1975$ & Montréal & Fl., pno \\
\hline
\end{tabular}

(4) Sur l'étiquette de la bande originale de cette œuvre, le titre Random Music lui est attribué.

Toutefois, l'écriture sur cette étiquette n'est pas celle de Vivier, mais d'un employé de l'Institut de sonologie. À notre avis, il s'agit ici plutôt d'une qualification que d'un véritable titre. 


\begin{tabular}{|c|c|c|c|}
\hline $\begin{array}{l}\text { 17. Pièce pour } \\
\text { violon et piano }\end{array}$ & $9-3-1975$ & [Montréal] & Vln, pno \\
\hline $\begin{array}{l}<18>\text { Pièce pour } \\
\text { violoncelle et } \\
\text { piano }<16-23>\end{array}$ & 1975 & [Montréal] & Vlc., pno \\
\hline $\begin{array}{l}<19>\text { Hymnen an } \\
\text { die Nacht } \\
<16-23>\end{array}$ & 1975 & [Montréal] & $\begin{array}{l}\text { Sop., } \\
\text { pno }\end{array}$ \\
\hline $\begin{array}{l}<20>\text { Pianoforte } \\
<16-23>\end{array}$ & 1975 & [Montréal] & Pno \\
\hline $\begin{array}{l}<21>\text { Pour guitare } \\
<16-23>\end{array}$ & 1975 & [Montréal] & Guitare \\
\hline $\begin{array}{l}<22>\text { Improvisation } \\
\text { pour basson et } \\
\text { piano }<16-23>\end{array}$ & 1975 & [Montréal] & Basson, pno \\
\hline 23. Liebesgedichte & $5-5-1975$ & Montréal & $\begin{array}{l}4 \text { voix mixtes, } \\
\text { vents }\end{array}$ \\
\hline $\begin{array}{l}\text { 24. Pour violon } \\
\text { et clarinette }\end{array}$ & 5-8-1975 & Halifax & $\begin{array}{l}\text { Vln, } \\
\text { clar. }\end{array}$ \\
\hline 25. Siddhartha & $28-2-1976$ & Ottawa & $\begin{array}{l}\text { Grand } \\
\text { orchestre }\end{array}$ \\
\hline $\begin{array}{l}\text { Prolifération } \\
\text { (rév.) }\end{array}$ & $24-4-1976$ & Montréal & $\begin{array}{l}\text { Ondes Martenot, } \\
\text { pno, perc. }\end{array}$ \\
\hline $\begin{array}{l}<26>\text { Learning } \\
<25-27>\end{array}$ & [1976] & & $\begin{array}{l}4 \text { vlns, } \\
\text { perc. }\end{array}$ \\
\hline 27. Woyzeck & c. $8-1976$ & [Montréal] & Bande \\
\hline $\begin{array}{l}\text { 28. Les } \\
\text { Communiantes }\end{array}$ & $22-1-1977$ & Montréal & Orgue \\
\hline $\begin{array}{l}<29>\text { Love } \\
\text { Songs }<29-32>\end{array}$ & 1977 & Montréal & $\begin{array}{l}\text { Ballet; } 7 \text { voix } \\
\text { mixtes }\end{array}$ \\
\hline
\end{tabular}




\begin{tabular}{|c|c|c|c|}
\hline $\begin{array}{l}<30>\text { Nanti } \\
\text { malam }<29-32>\end{array}$ & 1977 & [Montréal] & Ballet; 7 voix \\
\hline 31. Journal & 5-1977 & & $\begin{array}{l}4 \text { voix mixtes, } \\
\text { chœur SATB, } \\
\text { perc. }\end{array}$ \\
\hline 32. Pulau Dewata & 19-6-1977 & Montréal & $\begin{array}{l}\text { Ensemble } \\
\text { variable }\end{array}$ \\
\hline 33. Shiraz & $25-8-1977$ & Montréal & Pno \\
\hline $\begin{array}{l}<34>\text { Green } \\
<34-36>\end{array}$ & [1978] & & $\begin{array}{l}\text { Fl., vln, } \\
\text { vlc., pno }\end{array}$ \\
\hline 35. Paramirabo & $13-5-1978$ & Montréal & $\begin{array}{l}\text { Fl., vln, } \\
\text { vlc., pno }\end{array}$ \\
\hline 36. Greeting Music & $15-10-1978$ & Montréal & Petit ensemble \\
\hline 37. Kopernikus & $14-5-1979$ & Montréal & $\begin{array}{l}\text { Opéra en deux } \\
\text { actes }\end{array}$ \\
\hline 38. Orion & $6-10-1979$ & Montréal & Grand orchestre \\
\hline 39. Lonely Child & $5-3-1980$ & Montréal & Sop., ensemble \\
\hline 40. Zipangu & $13-8-1980$ & Montréal & 13 cordes \\
\hline $\begin{array}{l}\text { 41. Cinq Chansons } \\
\text { pour percussion }\end{array}$ & $26-9-1980$ & Montréal & Percussion \\
\hline $\begin{array}{l}\text { 42. Prologue pour } \\
\text { un Marco Polo }\end{array}$ & $1-3-1981$ & Montréal & $\begin{array}{l}5 \text { voix mixtes, } \\
6 \text { clar., } 2 \text { perc., } \\
\text { cordes, bande }\end{array}$ \\
\hline $\begin{array}{l}\text { 43. Wo bist du } \\
\text { Licht! }\end{array}$ & $11-3-1981$ & Montréal & $\begin{array}{l}\text { Mezzo-sop., } \\
\text { perc., cordes, } \\
\text { bande }\end{array}$ \\
\hline $\begin{array}{l}<44>\text { Bouchara } \\
<42-45>\end{array}$ & 1981 & & Sop., ensemble \\
\hline 45. Samarkand & $17-9-1981$ & Montréal & $\begin{array}{l}\text { Quintette ò } \\
\text { vents, pno }\end{array}$ \\
\hline
\end{tabular}




\begin{tabular}{|l|l|l|l|}
\hline $\begin{array}{l}\text { 46. Et je reverrai } \\
\text { cette ville étrange }\end{array}$ & 26-10-1981 & Montréal & Petit ensemble \\
\hline 47. A Little Joke & $2-12-1981$ & Montréal & Chœur SATB \\
\hline $\begin{array}{l}\text { 48. Trois Airs } \\
\text { pour un opéra } \\
\text { imaginaire }\end{array}$ & $3-11-1982$ & Paris & Sop., ensemble \\
\hline $\begin{array}{l}\text { 49. Glaubst du } \\
\text { an die Unsterb- } \\
\text { lichkeit der } \\
\text { Seele (inachevé) }\end{array}$ & $3-1983$ & Paris & $\begin{array}{l}\text { Chœur SATB, } \\
\text { 3 synth., 2 perc. }\end{array}$ \\
\hline
\end{tabular}

1. Quatuor à cordes

Pas de signature. Créé à Orford en août 1968, donc probablement composé en 1968 (catalogue de la Société de droits d'exécution - ci-après: S. D. E. ;édition de 1984(5)), à Montréal (où Vivier vivait et étudiait). Titre dans le manuscrit: "Quatuor à cordes \# 1 ». Un document signé Claude Vivier et se trouvant aux archives du Conservatoire de Montréal énumère les cinq œuvres suivantes: Quatuor à cordes, Ojikawa, Musique pour une liberté à bâtir, Prolifération, Hiérophanie. Vivier précise qu'il s'agit là de leur ordre chronologique.

\section{Ojikawa}

Pas de signature. Probablement composé en 1968 (catalogue S. D. E.) à Montréal.

\section{Musique pour une liberté à bâtir}

Pas de signature. Probablement composé en 1968 ou 1969 à Montréal. Dans le manuscrit, le titre est suivi du chiffre II. S'agit-il d'une deuxième version ou d'une suite à une autre œuvre?

\section{Prolifération}

Manuscrit non localisé. Probablement composé à Montréal, au plus tard en 1969, puisque la création a eu lieu en avril 1969. C'est aussi l'année mentionnée dans le catalogue de la S. D. E.

\section{Hiérophanie}

Signé «Montréal le 8/1/71». Une première version est signée "Heidelberg, août 1970", avec la remarque "ceci n'est pas la version définitive, c'est une première version!»

\section{Musik für das Ende}

Signé «Köln juli 71/ Amen!»

(5) Ce document n'a pas été considéré comme une source originale. Nous l'avons cité uniquement lorsqu'il concordait avec d'autres indications. 


\section{Deva et Asura}

Signé «Utrecht le 8 janvier 1972».

\section{Variation I}

Une composition inconnue auparavant, découverte par nous en juillet 1991. Bande originale à I'Institut de sonologie à La Haye ${ }^{(6)}$. Date de la copie stéréo: le 22 février 1972 (source : l'étiquette de la copie), donc probablement composé peu avant cette date à Utrecht, où se trouvait I'Institut à cette époque.

\section{9. (sans titre)}

Musique d'environnement composée en collaboration avec Peter Hamlin, dans le cadre d'un évènement multidisciplinaire sur le thème du hasard qui a eu lieu à l'université d'Utrecht. Bande originale à l'Institut de sonologie à La Haye. Date de composition: le 10 mars 1972 (source: l'étiquette de l'original), à Utrecht.

10. Hommage. Musique pour un vieux Corse triste

Bande originale à l'Institut de sonologie à La Haye. Date de la copie stéréo: le 8 mai 1972 (source: l'étiquette de la copie), donc probablement composé peu avant cette date à Utrecht.

\section{Désintégration}

Signé "Paris le 26 août 1972/Deo gratias».

\section{$<12>$ Chants}

Signé en bas de la première page « (C) Claude Vivier, 1972» et (à la fin) "Deo gratias». Composé à Cologne, probablement achevé en 1973 (catalogue S. D. E.). L'année inscrite dans la partition fait plutôt référence au début de la rédaction (décembre 1972): "Je venais d'arriver à Cologne, et je commençais d'étudier avec Karlheinz Stockhausen. [...] C'est donc en décembre 1972 que j'entrepris la rédaction de l'œuvre." (Texte de la pochette du disque Nocturnales, ici même p. 55.)

\section{$<13>0$ ! Kosmos}

Signé «Köln Den 13/2/73»

\section{$<14>$ Jesus erbarme dich}

Signé en bas de la première page « (C) Claude Vivier 1973». Fort probablement composé à Cologne dans le cadre des cours de composition de Stockhausen, comme $\mathrm{O}$ ! Kosmos, et plus ou moins simultanément avec celle-ci.

\section{Désintégration (rév.)}

Signé «1974 3 April Köln»
(6) Nous soulignerons cette découverte, ainsi que l'importance de l'œuvre, dans une publication ultérieure sur la production électroacoustique de Vivier. 
15. Lettura di Dante

Signé «Deo Gratias/Köln den 16/7/74».

16. Pièce pour flûte et piano

Signé «Montréal le 16/2/75». Les œuvres 16 à <22> inclusivement ont été composées pour la finale du Concours de musique du Canada, section Tremplin international, qui a eu lieu le 22 juin 1975. Le concours a commandé sept œuvres pour les interprètes finalistes. Vivier les a remises au fur et à mesure à raison d'une par mois environ entre décembre 1974 et mai 1975. Seulement trois ont été signées avec une date précise. Un problème se pose concernant Pièce pour violon et clarinette (voir sous 24).

17. Pièce pour violon et piano

Signé «9/3/75».

$<18>$ Pièce pour violoncelle et piano $<16-23>$

Signé «1975».

<19> Hymnen an die Nacht <16-23>

Signé «(C) Claude Vivier 1975».

$<20>$ Pianoforte $<16-23>$

Signé «(1975)».

$<21>$ Pour guitare <16-23>

Signé «1975».

$<22>$ Improvisation pour basson et piano $<16-23>$

Pas de signature.

23. Liebesgedichte

Signé «Montréal le 5 mai». Nous avons pu identifier l'année de composition 1975 à partir, entre autres, des documents suivants: Claude Vivier, lettre à Harry Halbreich du 12 août 1975; Claude Gingras, "La Société de musique contemporaine représentera le Québec à Washington", La Presse, 9 septembre 1975. Figurant à la première page de la partition, "(C) 1976» a toujours été cité comme année de composition, mais en fait il s'agit de l'année du dépôt légal.

24. Pour violon et clarinette

Signé «Halifax le 5/8/75». Le titre de cette œuvre figure sur une liste disant qu'elle a été ou devait être composée pour le concours mentionné sous 15 (archives du Concours de musique du Canada). Toutefois, la date de signature est postérieure à la période à laquelle s'est déroulé le concours. De surcroît, l'ex-directeur et fondateur du concours, M. Claude Deschamps, 
affirme que jamais il n'a commandé une pièce pour ces deux instruments. (Communication personnelle, mai 1991.)

\section{Siddhartha}

Signé "ottawa le 28 Février». L'année de composition est 1976, ce que nous avons déduit, entre autres, de l'affirmation suivante: "Je viens justement de terminer ma pièce pour Orchestre! Elle se nomme "Siddhartha" [...]» (Claude Vivier, lettre à Harry Halbreich, 10 mars 1976, Ottawa).

Prolifération (rév.)

Signé "Montréal le 24 avril 76». Le fait que Vivier, et d'autres après lui, ait donné 1975 comme année de révision s'explique probablement par la signature du solo pour piano («10/3/75»); qui constitue la première étape de la révision.

$<26>$ Learning $<25-27>$

Manuscrit non localisé. D'après le catalogue de la S. D. E., composé en 1976.

$<27>$ Woyzeck

Musique pour une production de théâtre de marionnettes du Centre national des arts. Bande originale non localisée. Composé entre le 13 juillet, date de la confirmation de la commande, et le 6 septembre 1976, date du début des répétitions. (Jean Herbiet, lettre à Claude Vivier, 13 juillet 1976, Ottawa.)

\section{Les Communiantes}

Signé «Montréal le 22 janv. 77»

$<29>$ Love Songs <29-32>

Signé en première page «(C) Claude Vivier, Montréal (1977)». Composé dans la même période que Nanti malam. (Jean-Pierre Perreault, communication personnelle, mai 1991.)

$<30>$ Nanti malam <29-32>

Pas de signature. Composé avant le 30 juillet 1977, date d'un article sur le spectacle (A. M. Ashley, "Dance for the Senses, but not the Intellect», The Citizen, 30 juillet 1977.)

31. Journal

Signé «may 1977».

32. Pulau Dewata

Signé «Montréal le 19/6/77».

33. Shiraz

Signé «Montréal le 25/8/77». 
$<34>$ Green $<33-35>$

Manuscrit non localisé. Probablement composé en 1978 (catalogue S. D. E.).

35. Paramirabo

Signé «Montréal le 13 mai 78 ».

36. Greeting Music

Manuscrit non localisé. Signature de Vivier reprise par la copiste sur le matériel: «Montreal, october 15th, 1978».

37. Kopernikus

Signé «Montréal le 14 mai 79/ Deo gratias». L'année 1980, toujours citée comme année de composition, par Vivier et autres, est l'année de création.

38. Orion

Signé «Montréal le 6 octobre 1979».

39. Lonely Child

Signé «Montréal le 5 mars 1980».

40. Zipangu

Signé "Montréal le 13 aout 1980».

41. Cinq Chansons pour percussion

Signé "Montréal le 26 sept 1980».

42. Prologue pour un Marco Polo

Signé «Montréal le $1^{\text {er }}$ mars 1981 ».

43. Wo bist du Licht!

Signé "Montréal le 11 mars 1981 ».

$<44>$ Bouchara $<42-45>$

Signé « 1981 ». Antérieur à Et je reverrai cette ville étrange, parce que Vivier mentionne dans une entrevue plusieurs de ses œuvres, dont Bouchara, et affirme ensuite que Et je reverrai cette ville étrange est la toute dernière. (S. Frykberg, "Claude Vivier in Conversation», Musicworks 1982, vol. 18, pp. 8-9.)

45. Samarkand

Signé «Montréal le 17 sept 1981 ».

46. Et je reverrai cette ville étrange

Signé «Montréal le 26 oct. 1981 ». 
47. A Little Joke

Signé «Montréal le 2 Décembre 1981».

48. Trois Airs pour un opéra imaginaire

Signé "Paris le 3 novembre 1982».

49. Glaubst du an die Unsterblichkeit der Seele

Inachevé, pas de signature. La rédaction de la partition a été interrompue à Paris, en mars 1983. Le titre sous lequel l'œuvre est connue, Crois-tu en l'immortalité de l'âme, est la traduction de celui du manuscrit. 


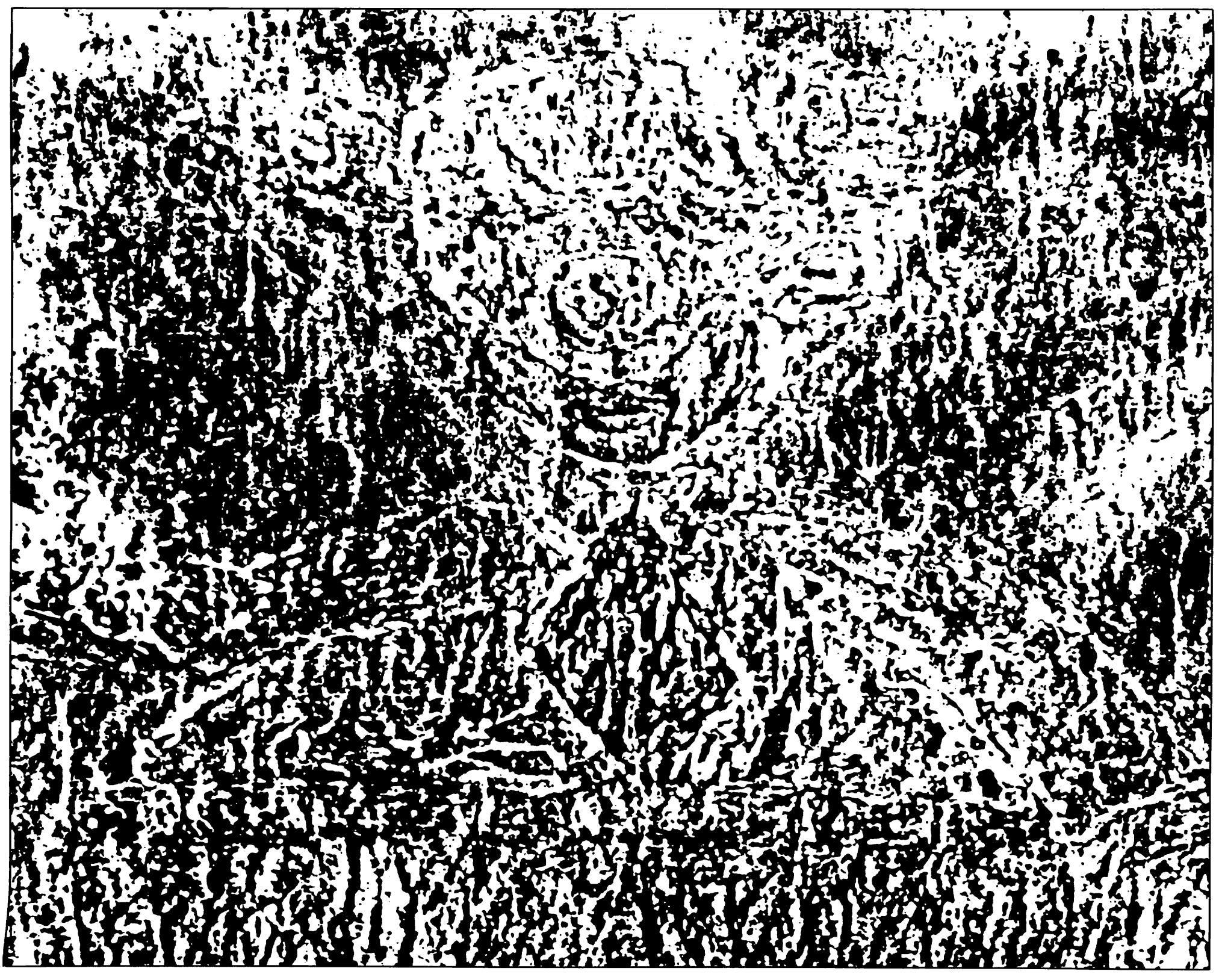

